\title{
Cranial Ultrasonography in Infantile Encephalitic Beriberi: A Useful First-Line Imaging Tool for Screening and Diagnosis in Suspected Cases
}

\author{
(D) N.A. Wani, (D).A. Qureshi, DK. Ahmad, and (D) N.A. Choh
}

\begin{abstract}
BACKGROUND AND PURPOSE: Brain imaging is central to the diagnosis of infantile encephalitic beriberi. Because cranial sonography findings have not been described in infantile encephalitic beriberi, our aim was to investigate its role in the diagnosis of this condition.
\end{abstract}

MATERIALS AND METHODS: We performed a retrospective review of head sonography of infants (admitted between November 1, 2014, and March 31, 2015) who presented with encephalopathy. Cranial ultrasonography scans were studied for the alteration of echogenicity of the basal ganglia.

RESULTS: Of the 145 consecutive infants who presented with encephalopathy, 58 had thiamine-responsive encephalopathy (infantile encephalitic beriberi) and 87 had encephalopathy due to other causes. Forty-eight of 145 infants with encephalopathy showed hyperechoic basal ganglia. A hyperechoic appearance of the basal ganglia on cranial ultrasonography was found to have a sensitivity of $71 \%$ (41/58) and a specificity of $92 \%$ (80/87) in diagnosing infantile encephalitic beriberi. The sensitivity of cranial sonography increased with age. It was a maximum of $93 \%(14 / 15)$ in the 5 months and older age group. Specificity was a maximum of $100 \%(18 / 18)$ in infants older than 5 months of age. Sensitivity was maximum in Wernicke encephalopathy at $90 \%(18 / 20)$ and least in the acidotic form at $43 \%(10 / 23)$. Follow-up showed gradual normalization of the hyperechoic appearance of the basal ganglia during 8 weeks in 26/41 (63\%), with mild atrophy of the basal ganglia in 6/41 (15\%)

CONCLUSIONS: Hyperechogenicity of the basal ganglia on cranial ultrasonography is a sensitive finding for the diagnosis of infantile encephalitic beriberi in infants who present with Wernicke encephalopathy.

ABBREVIATIONS: cUS = cranial ultrasonography; IEBB = infantile encephalitic beriberi

$\mathrm{T}$ hiamine deficiency disease is called beriberi and may have neurologic (dry beriberi) or cardiovascular (wet beriberi) manifestations. Thiamine deficiency is found in alcoholics and rice-eating populations consuming polished rice. ${ }^{1}$ Wernicke encephalopathy, characterized by ataxia, confusional state, and ocular motor abnormalities, is an acute neurologic manifestation of beriberi that can be seen in adults and children. Neurologic manifestations of thiamine deficiency in infants are nonspecific and include encephalopathy, vomiting, nystagmus, ptosis, moaning, and convulsions-infantile encephalitic beriberi (IEBB). Infants may develop beriberi, including its encephalitic form, if they are

Received November 14, 2015; accepted after revision January 20, 2016.

From the Departments of Pediatric Radiology (N.A.W.) and Pediatrics (U.A.Q., K.A.), Government Medical College, Srinagar, Jammu and Kashmir, India; and Department of Radiology (N.A.C.), SheriKasmir Institute of Medical Sciences, Srinagar, Jammu and Kashmir, India.

Please address correspondence to Nisar A. Wani, MD, Department of Pediatric Radiology, Govt GB Pant Children Hospital Sonwar, Srinagar Pin-190016, Jammu and Kashmir, India; e-mail: ahmad77chinar@gmail.com

http://dx.doi.org/10.3174/ajnr.A4756 exclusively breastfeeding from thiamine-deficient mothers. Rapid diagnosis of infantile encephalitic beriberi is essential to prevent mortality and neurologic sequelae. Imaging can facilitate early diagnosis of IEBB. ${ }^{1-4}$

The infant brain can be imaged with CT, MR imaging, and cranial ultrasonography (cUS). Use of CT for imaging the infant brain is associated with the radiation-related risks due to use of ionizing radiation and iodinated contrast agents. Apart from these risks, limited spatial resolution of CT for imaging of the infant brain is an additional disadvantage. ${ }^{5-8}$ Neuroimaging with MR imaging is considered optimal for the diagnosis of IEBB., However, MR imaging requires transfer of sick infants from the intensive care unit to the imaging center and frequently requires sedation before imaging. ${ }^{6-9}$ In addition, MR imaging may not be available to all in developing countries. Cranial ultrasonography is free from radiation hazards, less expensive than MR imaging, and often widely available, even in the developing countries. cUS is portable and can be performed rapidly at the bedside. ${ }^{5,9,10}$ cUS can be repeated easily if and when deemed necessary without 
compromising patient safety. MR imaging findings have been documented in IEBB, but there is no previous study describing cranial sonography findings in IEBB, to our knowledge. The present study aimed to evaluate the role of cUS in the diagnosis of IEBB.

\section{MATERIALS AND METHODS}

A retrospective review of cranial ultrasonography of all infants who were admitted between November 1, 2014, and March 31, 2015 with encephalopathy at our institution was performed. The study was approved by the institutional review board. A waiver of informed consent was granted for this retrospective analysis.

Encephalopathy had been diagnosed by the presence of 2 of the following symptoms: altered state of consciousness, seizures, and altered personality or cognition. All of these children had been investigated for the cause of encephalopathy. Laboratory investigations included complete blood count, serum biochemistry and electrolyte levels, blood sugar levels, and blood gases. CSF examination had been done at the discretion of the treating physician. Blood ammonia levels, tandem mass spectrometry, and urine gas chromatography-mass spectrometry had been performed to rule out inborn errors of metabolism.

Thiamine had been given at admission as a 100-mg intravenous infusion in all the infants with encephalopathy.

Infants were divided into 2 groups: 1) those who had encephalopathy due to thiamine deficiency, labeled IEBB; and 2) those who had a diagnosis other than IEBB.

The diagnosis of thiamine deficiency had been made by a dramatic response to thiamine (in all) and low blood thiamine levels (in 7). Detailed epidemiologic, dietary, clinical, laboratory, and treatment data of these children had been obtained.

Cranial ultrasonography had been performed with the Acuson X300 scanner (Siemens Medical Solutions, Malvern, Pennsylvania) by using the anterior fontanelle as the window, by a pediatric radiologist (N.A.W.) or sonologist with 3-5 years' experience performing head sonography in infants. A multifrequency (3-7.5 $\mathrm{MHz}$ ) phased array (sectoral) transducer with a small footprint had been used for scanning the brain parenchyma in the coronal and sagittal planes. All infants had the first cUS at initial presentation. Repeat scans were performed 2-3 weeks after the initial presentation and serially thereafter at intervals of 2-4 weeks.

Archived cUS studies were independently interpreted in retrospect by 2 radiologists with 5 and 8 years' experience in interpreting head sonography. The radiologists who interpreted the cUS studies were blinded to the clinical diagnosis and reviewed sonograms for normal anatomy and focal or diffuse altered echogenicity of brain parenchyma, with particular focus on deep gray matter structures. The basal ganglia were labeled as hyperechoic when their echogenicity was comparable with that of the choroid plexus or slightly less; and they were labeled mildly hyperechoic when the echogenicity was more than that of gray matter and comparable with that of periventricular white matter. The presence of ventricular dilation and any space-occupying lesion was sought on cUS. Initial findings were serially assessed in the follow-up studies.

\section{Statistical Analysis}

The accuracy of cUS was assessed by calculating sensitivity and specificity. Comparison of proportions and percentages was performed by applying $\chi^{2}$ and Fisher exact tests. All significant variables on univariate analysis were subjected to multivariate analysis. Agreement between 2 radiologists was calculated with linear weighted $\kappa$ by using VassarStats: Website for Statistical Computation (http://www.vassarstats.net/). Ninety-five percent confidence intervals were calculated. Statistical software, SPSS 20 (IBM, Armonk, New York), was used for all other data analysis. $P$ values $<.05$ were considered significant.

\section{RESULTS}

One hundred forty-five consecutive infants with encephalopathy were included in the study. Fifty-eight of 145 (40\%) had IEBB, and $87 / 145(60 \%)$ had encephalopathy due to causes other than IEBB.

Forty-one infants $(41 / 58)$ with IEBB had positive findings on cUS. There were 13 male and 28 female infants in the age range of 35 days to 9 months (mean age, 3.5 months). Consanguinity was present in 4 , and sibling death due to similar illness, in 2 . All the infants were exclusively breastfed by mothers, $29 / 41(71 \%)$ of whom resided in rural areas. Complementary diet (solid food introduced after 6 months) was not started in 12 infants despite being at least 6 months of age. Maternal staple diet consisted of polished rice consumed after washing it multiple times. As a part of cultural practice, 28/41 (68\%) lactating mothers consumed a diet that was restricted to soup derived from boiling meat with rice. We noticed 3 patterns: 1- to 2-month-old infants (16/41) presented with lactic acidosis. Infants older than 5 months of age (11/41) presented with Wernicke encephalopathy. The intermediate group (14/41) had mixed features of acidosis and Wernicke encephalopathy.

Clinical and laboratory features of infants in the IEBB group with positive findings and negative findings on cUS are compared in the Table. There was no statistically significant difference in individual clinical features between the 2 subgroups.

Forty-one of 58 (71\%) infants with IEBB had symmetric hyperechoic basal ganglia on cUS, and 17/58 (29\%) had normal findings on cUS. Seven of 87 (8\%) infants with encephalopathy due to other reasons had symmetric hyperechoic basal ganglia, and $80 / 87$ (92\%) had normal findings on cUS or findings other than hyperechoic basal ganglia (Fig 1).

Forty-eight of 145 infants with encephalopathy showed hyperechoic basal ganglia. Forty-one of $48(85 \%)$ with hyperechoic basal ganglia had IEBB, and $17 / 97$ (17.5\%) with nonhyperechoic basal ganglia had IEBB. A hyperechoic appearance of the basal ganglia on cUS was found to have a sensitivity of $71 \%(41 / 58 ; 95 \%$ CI, $57.99 \%-80.82 \%)$ and a specificity of $92 \%(80 / 87 ; 95 \% \mathrm{CI}$, $84.31 \%-96.05 \%)$ in diagnosing IEBB. The sensitivity of head ultrasonography increased with age. It was 55\% (16/29; 95\% CI, $37.55 \%-71.59 \%), 78 \%$ (11/14; 95\% CI, 52.41\%-92.43\%), and $93 \%(14 / 15 ; 95 \%$ CI, $70.18 \%-98.81 \%)$ in the age groups of $1-2$ months, 3-4 months, and 5 months and older, respectively. The specificity of head ultrasonography was $91 \%(40 / 44 ; 95 \% \mathrm{CI}$, $78.8 \%-96.4 \%)$ in the age group of $1-2$ months, $88 \%(22 / 25 ; 95 \%$ CI, $70.9 \%-95.8 \%$ ) in the age group of 3-4 months, and $100 \%$ 


\begin{tabular}{|c|c|c|c|}
\hline \multicolumn{4}{|c|}{$\begin{array}{l}\text { Clinical features of infants with IEBB with hyperechoic basal } \\
\text { ganglia }(n=41) \text { and normal basal ganglia }(n=17) \text { on HUS, with } \\
\text { univariate analysis showing the relationship between different } \\
\text { variables and the presence of hyperechoic basal ganglia on HUS }\end{array}$} \\
\hline & $\begin{array}{c}\text { Hyperechoic BG } \\
\text { (No.) (\%) }\end{array}$ & $\begin{array}{c}\text { Normal BG } \\
\text { (No.) (\%) }\end{array}$ & $P$ Value \\
\hline \multicolumn{4}{|l|}{ Age } \\
\hline $1-2 \mathrm{mo}$ & $16(39 \%)$ & $13(76 \%)$ & .019 \\
\hline $3-4 \mathrm{mo}$ & $11(27 \%)$ & $3(18 \%)$ & \\
\hline$\geq 5 \mathrm{mo}$ & $14(34 \%)$ & $1(6 \%)$ & \\
\hline \multicolumn{4}{|l|}{ Sex } \\
\hline Male & $13(32 \%)$ & $7(41 \%)$ & .551 \\
\hline Female & $28(68 \%)$ & $10(59 \%)$ & \\
\hline \multicolumn{4}{|l|}{ Clinical symptom complex } \\
\hline WE & $18(44 \%)$ & $2(12 \%)$ & .001 \\
\hline Mixed & $13(32 \%)$ & $2(12 \%)$ & \\
\hline Acidosis & $10(24 \%)$ & $13(76 \%)$ & \\
\hline \multicolumn{4}{|l|}{ Clinical features } \\
\hline \multicolumn{4}{|l|}{ Systemic } \\
\hline Fever & $11(27 \%)$ & $3(18 \%)$ & .523 \\
\hline Vomiting/reflux & $21(51 \%)$ & $12(70 \%)$ & .247 \\
\hline Diarrhea & 5 (12\%) & $3(18 \%)$ & .681 \\
\hline Decreased feeding & $16(39 \%)$ & $5(29 \%)$ & .560 \\
\hline Constipation & $1(2 \%)$ & $0(0 \%)$ & $>.999$ \\
\hline \multicolumn{4}{|l|}{ CNS } \\
\hline Altered sensorium & $41(100 \%)$ & 17 (100\%) & \\
\hline Irritability & $22(43)$ & $12(70 \%)$ & .260 \\
\hline Lethargy & $8(19 \%)$ & $2(12 \%)$ & .707 \\
\hline Moaning & $29(71 \%)$ & $14(82 \%)$ & .514 \\
\hline Vacant stare & $16(39 \%)$ & $3(18 \%)$ & .137 \\
\hline Ptosis & $18(44 \%)$ & $2(12 \%)$ & .032 \\
\hline Divergent squint & $13(32 \%)$ & $2(12 \%)$ & .188 \\
\hline Gross motor delay & $2(5 \%)$ & $0(0 \%)$ & $>.999$ \\
\hline Motor regression & $1(2 \%)$ & $0(0 \%)$ & $>.999$ \\
\hline Seizures & $15(36 \%)$ & $3(18 \%)$ & .217 \\
\hline Tonic posturing & $10(24 \%)$ & $3(18 \%)$ & .736 \\
\hline Hypotonia & $2(5 \%)$ & $0(0 \%)$ & $>.999$ \\
\hline \multicolumn{4}{|l|}{ CVS } \\
\hline Shock & $19(46 \%)$ & $14(82 \%)$ & .019 \\
\hline Tachycardia & $18(44 \%)$ & $15(88 \%)$ & .003 \\
\hline \multicolumn{4}{|l|}{ Metabolic } \\
\hline Acidotic breathing & $10(24 \%)$ & $13(76 \%)$ & $<.001$ \\
\hline
\end{tabular}

Note:-HUS indicates head ultrasonography; CVS, cardiovascular system; BG, basal ganglia; WE, Wernicke encephalopathy.
(18/18; 95\% CI, 78.47\%-100\%) in infants older than 5 months of age. The sensitivity also varied with clinical presentation. The sensitivity was maximum in Wernicke encephalopathy, 90\% (18/20; 95\% CI, 69.9\%-97.2\%), and least in acidotic forms, 43\% (10/23; $95 \%$ CI, $25.63 \%-63.19 \%)$. The specificity was similar, 92\% (80/ 87; 95\% CI, 84.31\%-96.05\%) in Wernicke encephalopathy, mixed and acidotic forms.

Univariate analysis (Table) showed that in IEBB, infants in the upper age groups, presenting with Wernicke encephalopathy and without shock, had a statistically significant presence of hyperechoic basal ganglia. Sex, standard base excess, and seizures were not significantly associated with this condition.

Multivariate logistic regression analysis showed that clinical presentation was an independent risk factor for positive findings of hyperechoic basal ganglia on cranial sonography (Wernicke encephalopathy: $\mathrm{OR}=9.810 ; 95 \% \mathrm{CI}, 1.085-88.687$; mixed: $\mathrm{OR}=7.560$; 95\% CI, 1.113-51.335). Shock was not an independent factor in depicting the positive findings on sonography $(\mathrm{OR}=0.778$; 95\% CI, 0.110-5.643).

\section{cUS Imaging}

The basal ganglia showed a bilaterally symmetric hyperechoic appearance in 41 infants with IEBB in the initial scan at presentation. Hyperechoic putamina (Figs $2 A$ and $3 A,-B$ ) were seen in $41 / 41$ $(100 \%)$ infants. The caudate nuclei appeared hyperechoic (Figs $3 A,-B$ and $4 A,-B)$ in $31 / 41$ (76\%); an isolated hyperechoic appearance of the bilateral putamen was seen in 10/41 (24\%). Slight hyperechogenicity of the bilateral median thalami (Fig 5) was seen in $3 / 41(7 \%)$. None of the scans showed any midline shift or mass lesion/hemorrhage. Seven infants with encephalopathy other than IEBB had hyperechoic basal ganglia on head ultrasonography. Bilateral putamina were hyperechoic in $7 / 7$ (100\%); caudate nuclei, in 5/7 (71\%); and thalami, in 4/7 (57\%).

Mild increased echogenicity of the basal ganglia was noted in infants 1-2 months of age, comprising 16/41 (39\%) cases with positive findings of IEBB on cUS. Children older than 2 months of age, comprising 25/41 (61\%) cases positive for IEBB, showed a distinctly hyperechoic appearance of the basal ganglia. Twelve of $16(75 \%)$ infants younger than 2 months of age showed hyperechoic putamina and caudate nuclei, and 4/16 (25\%) had hyperechoic putamina only. Nineteen of 25 (76\%) infants older than 2 months of age showed hyperechoic putamina and caudate nuclei, and 6/25 (24\%) had hyperechoic putamina only. There was no correlation between the age of the children and the relative involvement of the putamen and caudate nucleus $\left(\chi^{2}=\right.$ 0.09, $P=.764)$.

Follow-up scans showed regression of basal ganglia hyperechogenicity (Fig $2 B$ ) after thiamine administration, with almost normal appearance of basal ganglia in 2-4 weeks in 18/41; in 4-8 weeks, an additional $8 / 23$ children showed resolution of basal ganglia hyperechogenicity, totaling 26/41 (63\%) infants. Twenty-two of 26 infants with resolu-

FIG 1. Breakdown of infants with encephalopathy. "Hyperechoic BG" indicates CUS finding of increased echogenicity of the bilateral basal ganglia. "Normal" indicates CUS showing normal brain parenchyma and basal ganglia echogenicity. "Other findings" indicates cUS abnormalities other than hyperechoic basal ganglia-like hydrocephalus or a mass lesion. 

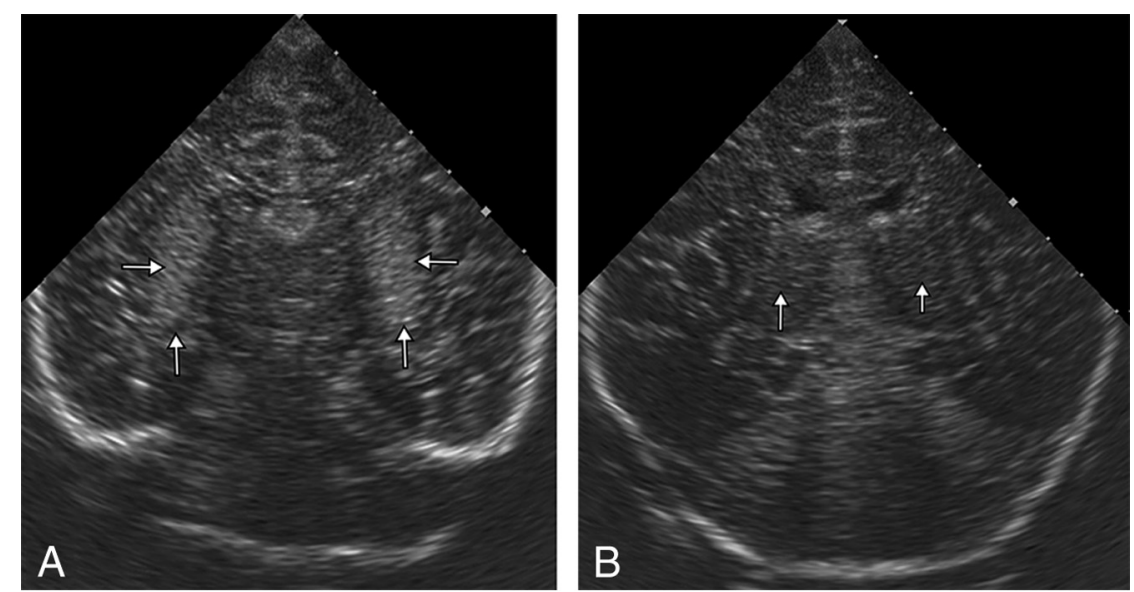

FIG 2. A 3-month-old girl with encephalopathy, reflux, and ptosis due to IEBB. A, Coronal plane cUS shows a bilaterally symmetric hyperechoic putamen (arrows). B, Follow-up cUS (after thiamine supplementation) in the coronal plane 6 weeks later shows very minimal hyperechogenicity in the putamen (arrows). Hyperechogenicity has regressed compared with the initial cUS (A).
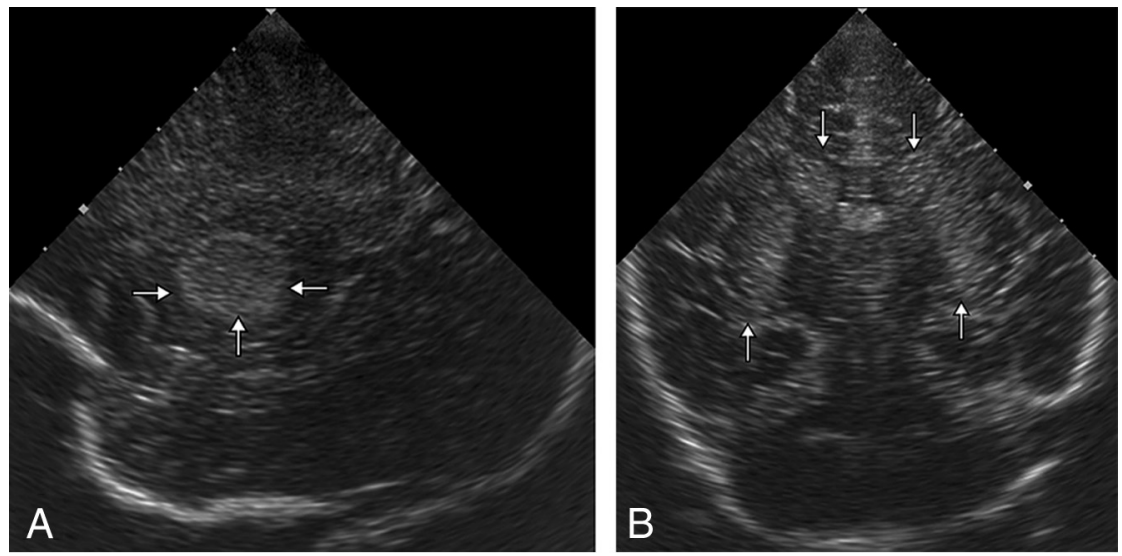

FIG 3. A 3-month-old girl with encephalopathy, reflux, and ptosis due to IEBB. A, Sagittal plane cUS shows a hyperechoic putamen (arrows). $B$, Coronal plane cUS shows a bilaterally symmetric hyperechoic caudate nucleus (downward arrows) and putamen (upward arrows).
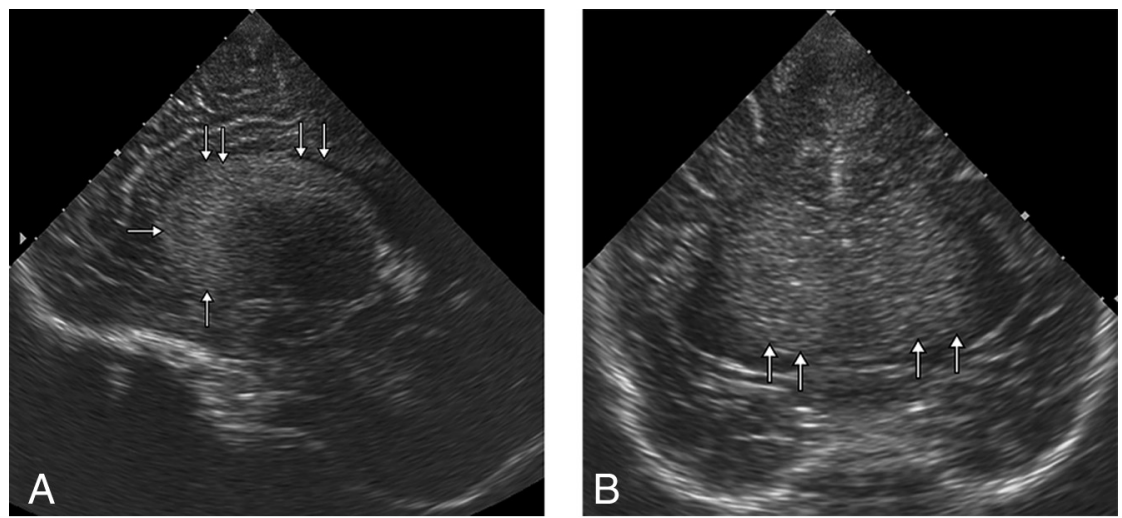

FIG 4. A 4-month-old boy with encephalopathy, moaning, and seizures diagnosed as IEBB. A, Sagittal plane CUS shows a curvilinear hyperechoic structure representing the caudate nucleus (arrows). B, Coronal plane cUS shows a bilateral symmetric hyperechoic caudate head (arrows).

tion of basal ganglia hyperechogenicity achieved developmental milestones at an appropriate age, and 4/26 showed motor delay at follow-up. Fifteen of 41 (37\%) infants did not have normalization of basal ganglia echogenicity at follow-up; 4/15 were followed for
$<4$ weeks. Ten children (10/15) with persistent basal ganglia hyperechogenicity showed delayed developmental milestones. Mild ventricular dilation of the lateral ventricles was seen in 6/41 (15\%). Three children (3/6) showed ventricular dilation at 2-4 weeks, and $3(3 / 6)$ showed ventriculomegaly after 4 weeks.

The symptomatic response to thiamine in IEBB was dramatic with moaning and tachycardia subsiding within 4 hours, and vacant stare and ptosis, in 6 hours. Lethargy persisted for 24 hours. In patients with developmental delay, milestones and tone were slow to recover during 4-14 weeks. Normal breastfeeding was achieved in a mean of 6 hours. The mean duration of the hospital stay was 3.25 days. At discharge, patients were put on 6 weeks of daily thiamine, with dietary advice to the mothers.

\section{DISCUSSION}

Early and rapid diagnosis of IEBB is essential so that thiamine is administered in time to prevent permanent brain damage and neurologic morbidity in the child. ${ }^{11,12}$ Thiamine levels can be determined in body fluids, but the procedure is time-consuming and may not be available to all. MR imaging of the brain is considered optimal for a rapid imaging-based diagnosis of IEBB. ${ }^{3,4,13}$

Several studies and case reports have documented abnormal findings on brain MR imaging in infants with IEBB. ${ }^{3,4,13}$ MR imaging in IEBB shows signal-intensity changes in the basal ganglia and cerebral cortex universally, besides changes in the medial thalami, periaqueductal gray matter, and mammillary bodies. Extrabasal ganglia changes (in the brain stem and mammillary bodies) are reported to be less frequent in the IEBB seen in the developing countries where symmetric T2WI hyperintense signal intensity involving the putamina alone or along with the caudate nuclei may be considered suggestive of the diagnosis in the presence of relevant clinical features. Altered-signalintensity lesions in the thalami and cerebral cortex are seen in a small proportion of children with IEBB. ${ }^{2,4}$ These lesions are always seen in the presence of basal ganglia lesions and are never seen in the absence of putaminal signal changes in IEBB. ${ }^{2,4}$

Ultrasonography can be used for imaging of the brain in infants, without risk of radiation exposure, and is readily available. 


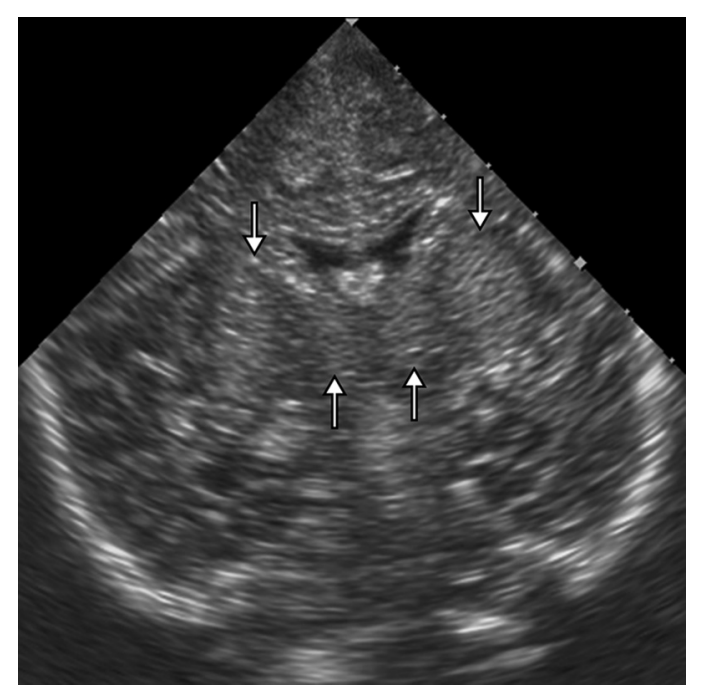

FIG 5. A 3-month-old boy with encephalopathy, ptosis, moaning, and reflux due to IEBB. Coronal plane cUS shows bilaterally symmetric hyperechoic putamen (downward arrows) and mildly hyperechoic thalami (upward arrows).

Cranial ultrasonography has been described as useful in conditions like hypoxic-ischemic injury and inborn errors of metabolism. However, there is no previous study describing cUS findings in IEBB, to our knowledge. This prompted us to undertake the present study.

Our study showed a hyperechoic appearance of the basal ganglia (putamen and caudate nucleus) on cUS in $71 \%$ of infants with IEBB. Putaminal hyperechogenicity was seen in all, and caudate hyperechogenicity was seen in $76 \%$ of the infants with abnormal findings on cUS. Putaminal and caudate changes on brain MR imaging are reported to be universally present in IEBB according to both Western and Indian literature. ${ }^{2,4,13}$ Studies from the thiamine-deficient Indian population report changes in the thalami, cerebral cortex, mammillary bodies, and brain stem in a smaller percentage of infants, always in the presence of basal ganglia lesions. We could not delineate cortical or brain stem changes in any infant, though a mild hyperechoic appearance of the thalami was seen in $7 \%$.

We have shown, in a sizeable cohort of infants with IEBB, that cUS detects main brain parenchymal changes that support the clinical diagnosis. Although some aspects of brain parenchymal changes are only or better seen with MR imaging, most salient features of basal ganglia involvement in IEBB were easily detected with cUS. The sensitivity and specificity of cUS were high for the diagnosis of IEBB in infants older than 5 months of age with features of Wernicke encephalopathy.

Hyperechoic basal ganglia on cUS in an infant with encephalopathy, metabolic acidosis, gastroesophageal reflux, and ptosis from the lower socioeconomic strata of developing countries suggest a diagnosis of IEBB. In the absence of blood thiamine levels and the availability of MR imaging, cUS may be useful in the triage of such infants. Infants older than 5 months of age with hyperechoic basal ganglia on cUS should always receive parenteral thiamine. cUS can rule out hydrocephalus, space-occupying lesionlike hemorrhage/abscess, or extra-axial collection as the cause and should be part of routine evaluation in such infants. ${ }^{14-18}$ The dif- ferential diagnosis of hyperechoic deep gray matter structures on cUS includes severe hypoxic-ischemic injury, inborn errors of metabolism, and encephalitis/congenital infection. ${ }^{5,10,19-22}$ Clinical features, serologic/biochemical investigations, and CSF analysis can help in making the proper diagnosis. ${ }^{2,23}$

The basal ganglia are metabolically very active in children having high concentrations of mitochondria and increased glucose use. Thiamine deficiency resulting in deficient adenosine triphosphate production consequent to defective thiamine-dependent oxidative glucose metabolism preferentially manifests with changes in the basal ganglia in children. ${ }^{4,11-13}$ Vasogenic and cytotoxic edema has been proposed as the underlying cause of basal ganglia changes in IEBB. An edematous appearance of the basal ganglia on cUS manifests with increased echogenicity. Basal ganglia changes in IEBB have been documented as reversible with early thiamine supplementation, and failure to administer thiamine may result in permanent brain damage. ${ }^{4,11,13}$

We were able to recognize basal ganglia lesions on cUS at presentation and continued with thiamine supplementation with favorable immediate clinical outcome in all. Basal ganglia hyperechogenicity was demonstrated to decrease slowly with thiamine, reverting to a normal appearance in 6-8 weeks in 63\% (26/41). In $37 \%$ (15/41) of children, the basal ganglia showed persistent hyperechogenicity, and $15 \%$ (6/41) showed some atrophy of the basal ganglia with ventriculomegaly in the follow-up.

\section{Limitations}

The low sensitivity of hyperechoic basal ganglia on cUS in infants younger than 2 months of age (55\%) and in infants who present with clinical acidosis (43\%) prevents its use as a screening tool for the diagnosis of IEBB in these patients. The suboptimal and inadequate visualization of changes in the thalami, brain stem, and mammillary bodies may limit the usefulness of cUS in cases in which changes are seen predominantly in these structures. An inadequate acoustic window through a closed anterior fontanelle makes cUS difficult in infants with craniosynostosis. Closure of the anterior fontanelle with time also limits the duration of longterm follow-up with cUS.

\section{CONCLUSIONS}

cUS is a preferable initial imaging technique for the infant brain because it is cheap and readily available, even in developing countries; is safe from radiation risk; and does not need transfer of infants and sedation before examination. Access across the anterior fontanelle is optimal in the age group afflicted with IEBB. Hyperechogenicity of the basal ganglia on cUS is a sensitive finding (90\%) for the diagnosis of IEBB in infants who present with Wernicke encephalopathy. Sensitivity is, however, low in those presenting with clinical acidosis (43\%). In the appropriate clinical settings, symmetric hyperechoic basal ganglia on cUS in an infant are also highly specific for the diagnosis of IEBB.

\section{REFERENCES}

1. Soukaloun D, Kounnavong S, Pengdy B, et al. Dietary and socioeconomic factors associated with beriberi in breastfed Lao infants. Ann Trop Paediatr 2003;23:181-86 Medline

2. Rao SN, Mani S, Madap K, et al. High prevalence of infantile enceph-

AJNR Am J Neuroradiol 37:1535-40 Aug 2016 www.ajnr.org

1539 
alitic beriberi with overlapping features of Leigh's disease. J Trop Pediatr 2008;54:328-32 CrossRef Medline

3. Zuccoli G, Siddiqui N, Bailey A, et al. Neuroimaging findings in pediatric Wernicke encephalopathy: a review. Neuroradiology 2010; 52:523-29 CrossRef Medline

4. Wani NA, Qureshi UA, Jehangir M, et al. Infantile encephalitic beriberi: magnetic resonance imaging findings. Pediatr Radiol 2016; 46:96-103 CrossRef Medline

5. Leijser LM, de Vries LS, Rutherford MA, et al. Cranial ultrasound in metabolic disorders presenting in the neonatal period: characteristic features and comparison with MR imaging. AJNR Am J Neuroradiol 2007;28:1223-31 CrossRef Medline

6. Lowe LH, Bailey Z. State-of-the-art cranial sonography, part 1: modern techniques and image interpretation. AJR Am J Roentgenol 2011;196:1028-33 CrossRef Medline

7. Wezel-Meijler Gv, de Vries LS. Cranial ultrasound: optimizing utility in the NICU. Curr Pediatr Rev 2014;10:16-27 CrossRef Medline

8. Hagmann CF, Robertson NJ, Acolet D, et al. Cranial ultrasound findings in well newborn Ugandan infants. Arch Dis Child Fetal Neonatal Ed 2010;9:F338-44 CrossRef Medline

9. Epelman M, Daneman A, Chauvin N, et al. Head ultrasound and MR imaging in the evaluation of neonatal encephalopathy: competitive or complementary imaging studies? Magn Reson Imaging Clin N Am 2012;20:93-115 CrossRef Medline

10. Yamagata T, Yano S, Okabe I, et al. Ultrasonography and magnetic resonance imaging in Leigh disease. Pediatr Neurol 1990;6:326-29 CrossRef Medline

11. Mimouni-Bloch A, Goldberg-Stern H, Strausberg R, et al. Thiamine deficiency in infancy: long-term follow-up. Pediatr Neurol 2014;51: 311-16 CrossRef Medline

12. Crook MA, Sriram K. Thiamine deficiency: the importance of recognition and prompt management. Nutrition 2014;30:953-54 CrossRef Medline
13. Kornreich L, Bron-Harlev E, Hoffmann C, et al. Thiamine deficiency in infants: MR findings in the brain. AJNR Am J Neuroradiol 2005; 26:1668-74 Medline

14. Yikilmaz A, Taylor GA. Sonographic findings in bacterial meningitis in neonates and young infants. Pediatr Radiol 2008;38:129-37 CrossRef Medline

15. van Leyen $\mathrm{K}$, Klötzsch C, Harrer JU. Brain tumor imaging with transcranial sonography: state of the art and review of the literature. Ultraschall Med 2011;32:572-81 CrossRef Medline

16. Fischer AQ. The use of ultrasound in evaluating neurologic diseases of childhood. Neurol Clin 1990;8:759-74 Medline

17. Babcock DS. Sonography of the brain in infants: role in evaluating neurologic abnormalities. AJR Am J Roentgenol 1995;165:417-23 CrossRef Medline

18. Simanovsky N, Taylor GA. Sonography of brain tumors in infants and young children. Pediatr Radiol 2001;31:392-98 CrossRef Medline

19. Fariello G, Dionisi-Vici C, Orazi C, et al. Cranial ultrasonography in maple syrup urine disease. AJNR Am J Neuroradiol 1996;17:311-15 Medline

20. Forstner R, Hoffmann GF, Gassner I, et al. Glutaric aciduria type I: ultrasonographic demonstration of early signs. Pediatr Radiol 1999; 29:138-43 CrossRef Medline

21. de Vries LS, Gunardi H, Barth PG, et al. The spectrum of cranial ultrasound and magnetic resonance imaging abnormalities in congenital cytomegalovirus infection. Neuropediatrics 2004;35:113-19 CrossRef Medline

22. de Vries LS, Verboon-Maciolek MA, Cowan FM, et al. The role of cranial ultrasound and magnetic resonance imaging in the diagnosis of infections of the central nervous system. Early Hum Dev 2006; 82:819-25 CrossRef Medline

23. Qureshi UA, Wani NA, Ahmad K, et al. Infantile Wernicke's encephalopathy. Arch Dis Child 2015;100:648 CrossRef Medline 